\title{
The response of Escherichia coli to exposure to the biocide polyhexamethylene biguanide
}

Correspondence
Andrew P. Morby
morby@cardiff.ac.uk

Received 31 October 2005

Revised 4 January 2006

Accepted 10 January 2006

\author{
Michael J. Allen,t Graham F. White and Andrew P. Morby \\ School of Biosciences, Cardiff University, Museum Avenue, PO Box 911, \\ Cardiff CF10 3US, UK
}

\begin{abstract}
The global response of Escherichia coli to the broad-spectrum biocide polyhexamethylene biguanide (PHMB) was investigated using transcriptional profiling. The transcriptional analyses were validated by direct determination of the PHMB-tolerance phenotypes of derivatives of $E$. coli MG1655 carrying either insertionally inactivated genes and/or plasmids expressing the cognate open reading frames from a heterologous promoter in the corresponding chromosomally inactivated strains. The results showed that a wide range of genes was altered in transcriptional activity and that all of the corresponding knockout strains subsequently challenged with biocide were altered in tolerance. Of particular interest was the induction of the rhs genes and the implication of enzymes involved in the repair/binding of nucleic acids in the generation of tolerance, suggesting a novel dimension in the mechanism of action of PHMB based on its interaction with nucleic acids.
\end{abstract}

\section{INTRODUCTION}

Polyhexamethylene biguanide (PHMB) is a broad-spectrum antibacterial agent that has been widely used for many years as an antiseptic in medicine and the food industry, and its current applications also include impregnation of fabrics to inhibit microbial growth (Cazzaniga et al., 2002; Payne \& Kudner, 1996); water treatment (Kusnetsov et al., 1997); disinfection of a variety of solid surfaces such as contact lenses (Hiti et al., 2002); as a mouthwash (Rosin et al., 2001, 2002); treatment of hatching eggs to prevent Salmonella infection (Cox et al., 1998, 1999); and as a treatment against fungi (Messick et al., 1999) and Acanthamoeba (Donoso et al., 2002; Gray et al., 1994; Narasimhan et al., 2002) in infective keratitis. Its preparations are mixtures of polymeric biguanides of structure $\left[-\left(\mathrm{CH}_{2}\right)_{6} \cdot \mathrm{NH} \cdot \mathrm{C}(=\mathrm{NH}) \cdot \mathrm{NH} \cdot \mathrm{C}(=\right.$ $\mathrm{NH}) \cdot \mathrm{NH}-]_{n}$ where $n=2-40$, with a mean size of $n=11$, giving a molecular mass range of approximately $400-8000$, with various combinations of amino $\left(-\mathrm{NH}_{2}\right)$, guanide $\left[-\mathrm{NH} . \mathrm{C}(=\mathrm{NH}) \cdot \mathrm{NH}_{2}\right]$ or cyanoguanide $[-\mathrm{NH} \cdot \mathrm{C}(=\mathrm{NH})$. $\mathrm{NH} . \mathrm{CN}]$ as end-groups.

PHMB is bacteriostatic at low concentrations (typically 1-10 $\mathrm{mg}^{-1}$ ), but bactericidal at higher concentrations, and inhibition of growth and bactericidal activity both increase with increased polymerization (Broxton et al., 1983; Gilbert

tPresent address: Plymouth Marine Laboratory, Prospect Place, The Hoe, Plymouth PL1 3DH, UK.

Abbreviation: PHMB, polyhexamethylene biguanide.

Original macroarray data have been deposited at the NCBI Gene Expression Omnibus (http://www.ncbi.nlm.nih.gov/projects/geo/index. cgi), accession number GSE2827. et al., 1990a). The lethal action is considered to involve interaction at the cytoplasmic membrane to cause nonspecific alterations in membrane integrity. The proposed basis for the polymer-size effect is that PHMB interacts with acidic membrane-lipids to cause phase separation and domain formation; larger PHMB molecules produce larger domains and therefore more disruption (Broxton et al., 1984; Ikeda et al., 1984). This view was refined by Gilbert et al. (1990b) who showed that although the activity increased with increasing length of the polymer, the effect of polymer length was much reduced above $n=6$. Comparison of whole cells and spheroplasts showed that the cell envelope, while not providing complete protection, provides a significant exclusion barrier. Removal of lipopolysaccharides from the outer envelope markedly increased the activity of highbut not low-molecular-mass fractions. These observations, and the discovery of a strong synergy between low- and high-molecular-mass components in biocidal activity, led Gilbert et al. (1990b) to conclude that the low-activity, lowmolecular-mass components enable larger homologues to gain access to sites of action in the cytoplasmic membrane.

Acanthamoeba castellanii treated with high concentrations of PHMB contained clusters of densely stained precipitates (Khunkitti et al., 1998). Moreover, PHMB treatment produced increased amounts of phosphorus inside the cells compared with untreated controls, and these accumulations were often confined to cell walls and nuclei (Khunkitti et al., 1999). Reduced membrane permeability causing retention of phosphorus, coagulation of proteins and aggregation of phospholipids have been considered as possible causes of elevated phosphorus but the possibility of association between PHMB and nucleic acids has not been 
considered hitherto, despite rapid growth in the literature in the last 20 years (Wallace, 2003) on the interaction of DNA with the natural polyamines, putrescine, spermine and spermidine (for a review, see Cohen, 1998), and with a variety of other synthetic polycationic compounds that are currently being developed as vehicles for non-viral transfection of DNA into cells for therapeutic purposes (Vijayanathan et al., 2002).

Recently, we showed that PHMB interacts strongly and cooperatively with nucleic acids in vitro and that the structural status of the nucleic acid affects the nature of its initial interactions with PHMB (Allen et al., 2004). Here we show that the interaction of PHMB with nucleic acids in vitro has a parallel in vivo, giving a novel facet to the action of PHMB. Using whole-genome transcriptional profiling, we show that the transcriptional rate of a wide range of genes in Escherichia coli strain MG1655 is altered by exposure of cells to PHMB, and that enzymes involved, inter alia, in the metabolism/repair of nucleic acids contribute to the tolerance of $E$. coli to bacteriostatic concentrations of PHMB. Phenotypic analyses in deletion mutants of strain MG1655 and in complemented deletions were used to confirm the biological significance of the results derived from transcriptional profiling.

\section{METHODS}

Bacterial strains and deletion mutants. Strains and plasmids are listed in Tables 1 and 2. E. coli K-12, strain MG1655 (F-, $\lambda^{-}$, rph-1) (Blattner et al., 1997) and its deletion mutants in which the genes aspA, flgJ, hdeA, hns, osmB, recA, rhsE and yebG had been interrupted by insertion of a kanamycin-resistance cassette, were obtained from the University of Wisconsin E. coli Genome Project (www.genome.wisc.edu). In addition, a cpxP-deletion mutant in E. coli $\mathrm{W} 3110\left[\mathrm{~F}^{-}, \lambda^{-}, \mathrm{IN}(r r n D-r r n E) r p h-1\right]$ (E. coli Genetic Stock Center, Yale University, CT, USA) bearing the pKD4/pKD46 plasmids (Table 1) was created in our laboratory using the method of Datsenko \& Wanner (2000) with the forward primer CATGACTTTA CGTTGTTTTA CACCCCCTGA CGCATGTTTG TGTAGGCTGG AGCTGC and reverse primer CTGACGCTGA TGTTCGGTTA AACTTATGCC GTCGAACATA TGAATATCCT CCTTAGTTC. Three PCR screens using locus-specific primers and the respective common test primer were used to verify the presence of both new junction fragments. A fourth PCR was carried out with both flanking locus-specific primers to verify the loss of the parental (non-mutant) fragment and gain of the new mutant fragment. DNA oligonucleotides were purchased from Sigma-Genosys or GibcoBRL. To allow phenotypic comparison with wild-type MG1655 and the Wisconsin mutants, the $c p x P$ gene deletion was transferred from strain W3110 to strain WG1655 by ultrasonic partial fragmentation of genomic DNA from the mutant strain, electroporation into MG1655 and screening for kanamycin resistance, using standard methods. The resulting MG1655 $\Delta c p x P$ mutant was verified using the PCR reactions described above. Parallel attempts to create knockout mutants in either $f r m B$ or $y c g W$ were unsuccessful, consistent with a report that these are essential genes (Gerdes et al., 2003). PHMB-resistance phenotypes of all deletion mutants were determined by measurement of minimum inhibitory concentration (MIC).

Overexpression plasmids. The Genome Analysis Project Japan (http://ecoli.aist-nara.ac.jp/) provides clones of each ORF predicted from the genome sequence of E. coli W3110 (Mori et al., 2000). Every ORF has been cloned into a plasmid (known as pCA24N) containing the IPTG-inducible promoter pT5/lac, an N-terminus histidine tag of the target ORF and an in-frame fusion of green fluorescent protein (GFP) at the C-terminus of the target. A cis-coded $\operatorname{lacl}^{q}$ is present to allow strict repression of the expression from the pT5/lac promoter (http://ecoli.aist-nara.ac.jp/gb5/Resources/archive/ archive.html). The fusion of GFP to a protein can have adverse effects upon protein folding, stability and function. Therefore, the GFP-encoding portion of the original pCA24N-based expression plasmid was removed using a Not I digest. The overexpression plasmids, previously known as pCA24N- $x x x X$ [The Genome Analysis Project Japan website (http://ecoli.aist-nara.ac.jp/)] with the GFP portion removed, are here referred to as pMJA- $x \times x X$ (Table 1). The plasmids were expressed in E. coli strain MG1655, and PHMBresistance phenotypes were determined by measurement of MIC.

Culturing conditions. Liquid cultures were grown in LB medium at $37^{\circ} \mathrm{C}$ with constant shaking at 200 r.p.m. unless stated otherwise. Growth was monitored by measuring optical attenuance at $600 \mathrm{~nm}$ $\left(D_{600}\right)$ in a Genequant Pro spectrometer (Amersham). For longterm storage, liquid cultures were mixed $1: 1(\mathrm{v} / \mathrm{v})$ with sterile glycerol, mixed thoroughly and stored at $-80^{\circ} \mathrm{C}$. To minimize PHMB adsorption to glass and thus to ensure reproducibility, all flasks used during bacterial growth experiments involving PHMB were washed in concentrated nitric acid, rinsed twice in distilled water, air-dried, rinsed with $2 \%$ dimethyldichlorosilane in 1,1,1-trichloroethane $(\mathrm{BDH})$, dried, baked at $130^{\circ} \mathrm{C}$ and then rinsed three times in distilled water.

PHMB. PHMB, kindly provided by Avecia (Manchester, UK), was a mixture of homologues with $n$ ranging from 2 to 15 and with a mean value of $5 \cdot 5$.

Table 1. E. coli $\mathrm{K}-12$ derivatives used in this study

\begin{tabular}{|lll|}
\hline Strain & \multicolumn{1}{c|}{ Genotype } & \multicolumn{1}{c|}{ Source } \\
\hline W3110 & $\mathrm{F}^{-} \lambda^{-} \mathrm{IN}(r r n D-r r n E), r p h-1$ & CGSC no. 4474 \\
MG1655 & $\mathrm{F}^{-} \lambda^{-} r p h-1$ & Blattner et al (1997) \\
MG1655 $\Delta a s p A$ & $\mathrm{~F}^{-} \lambda^{-} r p h-1 \Delta a s p A:: \mathrm{Km}$ & U. W. E. coli Genome Project \\
MG1655 $\Delta f l g J$ & $\mathrm{~F}^{-} \lambda^{-} r p h-1 \Delta f l g J:: \mathrm{Km}$ & U. W. E. coli Genome Project \\
MG1655 $\Delta h n s$ & $\mathrm{~F}^{-} \lambda^{-} r p h-1 \Delta h n s:: \mathrm{Km}$ & U. W. E. coli Genome Project \\
MG1655 $\Delta r h s E$ & $\mathrm{~F}^{-} \lambda^{-} r p h-1 \Delta r h s E:: \mathrm{Km}$ & U. W. E. coli Genome Project \\
MG1655 $\Delta$ osmB & $\mathrm{F}^{-} \lambda^{-} r p h-1 \Delta o s m B:: \mathrm{Km}$ & U. W. E. coli Genome Project \\
MG1655 $\Delta r e c A$ & $\mathrm{~F}^{-} \lambda^{-} r p h-1 \Delta r e c A:: \mathrm{Km}$ & U. W. E. coli Genome Project \\
\hline
\end{tabular}


Table 2. Plasmids used in this study

pMJA-based plasmids are IPTG inducible, containing His-tagged ORFs; see text for details.

\begin{tabular}{|lll|}
\hline Plasmid & \multicolumn{1}{c|}{ Relevant characteristics } & \multicolumn{1}{c|}{ Source } \\
\hline pKD46 & Recombination plasmid & Datsenko \& Wanner (2000) \\
pKD4 & Amplification of Km resistance & Datsenko \& Wanner (2000) \\
pCR-Blunt & Blunt-ended cloning vector & Invitrogen \\
pMJA & Overexpression plasmid (ORFless) & This study \\
pMJA- $a s p A$ & $a s p A$ overexpression plasmid & This study \\
pMJA- $f l g J$ & $f l g J$ overexpression plasmid & This study \\
pMJA- $h n s$ & $h n s$ overexpression plasmid & This study \\
pMJA- $o s m B$ & $o s m B$ overexpression plasmid & This study \\
pMJA-recA & $r e c A$ overexpression plasmid & This study \\
pMJA-rhsB & $r h s B$ overexpression plasmid & This study \\
pMJA-rhsD & $r h s D$ overexpression plasmid & This study \\
pMJA-rhsE & $r h s E$ overexpression plasmid & This study \\
pMJA- $y e b G$ & $y e b G$ overexpression plasmid & This study \\
pMJA- $y h a B$ & $y h a B$ overexpression plasmid & This study \\
\hline
\end{tabular}

Enzymes. Restriction endonucleases, DNA ligase, Vent and Taq polymerase supplied with their appropriate buffers were obtained from New England Biolabs. Bovine serum albumin (BSA), where required, was supplied with the enzyme. Deoxyribonuclease, free from ribonuclease, was obtained from Qiagen. M-MLV reverse transcriptase and reaction buffer were obtained from Promega.

Transcriptional profiling of $E$. coli using macroarrays. Samples of cell culture $(1 \mathrm{ml})$ were used to isolate total RNA using an RNeasy miniprep kit (Qiagen) according to the manufacturer's instructions. RNA was eluted in RNase-free water (Sigma). The RNA concentration was determined from $A_{260}$. The C-terminal primer set comprising 4290 ORF-specific C-terminal primers (SigmaGenosys) was used to generate hybridization probes in a standard first-strand cDNA synthesis. The Sigma-Genosys protocol was used to achieve $>60 \%$ incorporation of ${ }^{33} \mathrm{P}$ from $\left[\alpha-{ }^{33} \mathrm{P}\right] \mathrm{dCTP}$ (74-111 TBq $\mathrm{mmol}^{-1}$, NEN Life Science Products). Unincorporated nucleotides were removed by gel filtration through a MicroSpin G-25 Sephadex column (Amersham-Pharmacia) according to the manufacturer's instructions.

Hybridization of cDNAs to Panorama E. coli gene arrays (SigmaGenosys) and subsequent washing steps were carried out according to the manufacturer's instructions. Images representing the localization of hybridized probes were captured on a Personal Imager FX (Bio-Rad) using the PC-based Quantity One software. Arrays were stripped for reuse by washing at $100{ }^{\circ} \mathrm{C}$ with stripping solution as specified by the manufacturer.

Spot intensities on a given array were normalized by calculation of the intensity of each as a fraction of the total intensity of all spots taken together. The normalized intensities for each ORF in test (PHMBexposed) and control (unexposed) arrays were compared and the induction ratios (normalized intensity of test relative to that of the control) were calculated. Genes were considered to exhibit significantly changed expression if the $\log _{10}$ (induction ratio) was greater than 2 SD from the mean of the $\log _{10}$ (induction ratio) for all spots, in three separate experiments. Data are presented as fold-change of test and control (higher value divided by the lower) with positive and negative signs indicating induction and repression by PHMB, respectively. Original data have been deposited at the NCBI Gene Expression Omnibus (http://www.ncbi.nlm.nih.gov/projects/ geo/index.cgi), accession number GSE2827.
MIC. E. coli strains were assessed for tolerance to PHMB by growth in the presence of increasing concentration of PHMB to determine the MIC. They were grown for $18 \mathrm{~h}$ in LB broth $(5 \mathrm{ml}$, containing additions where appropriate, e.g. IPTG). Each culture was diluted in fresh LB broth (containing additions where appropriate) to an optical attenuance of precisely $0 \cdot 100$, then $145 \mu \mathrm{l}$ aliquots were dispensed into 96-well micro-titre plates containing PHMB ( $5 \mu \mathrm{l}$ per well) to give final concentrations ranging from $1 \cdot 25$ to $6 \cdot 75 \mathrm{mg} \mathrm{l}^{-1}$ in increments of $0.25 \mathrm{mg}^{-1}$. Plates were incubated at $37^{\circ} \mathrm{C}$, 200 r.p.m. for $48 \mathrm{~h}$ and growth monitored by measuring $D_{600}$. MIC was taken as the lowest concentration showing retarded growth. In all cases, triplicate assays were always in complete agreement (i.e. the same well in triplicate plates). The precision of the MIC values was therefore limited by the increment size and was taken as $\pm 0 \cdot 125 \mathrm{mg} \mathrm{l}^{-1}$.

\section{RESULTS}

\section{Effect of PHMB on the growth of E. coli}

At all concentrations tested $\left(2 \cdot 5-15 \mathrm{mg} \mathrm{l}^{-1}\right)$, addition of PHMB altered the growth characteristics of E. coli (Fig. 1). Concentrations $\geqslant 10 \mathrm{mg} \mathrm{l}^{-1}$ were bactericidal. The addition of $7.5 \mathrm{mg} \mathrm{PHMB}^{-1}$ at $D_{600} \sim 0.3$ caused a temporary cessation in growth lasting approximately $4-5 \mathrm{~h}$, after which growth resumed. The effect of the addition of $7 \cdot 5 \mathrm{mg}$ PHMB $1^{-1}$ to rapidly growing early exponential $E$. coli cultures on the shape of the growth curve was reproducible (see Figs 1 and 2) and this system was used as the basis for further experiments.

\section{DNA macroarray-based global transcription profiling of $E$. coli in response to PHMB treatment}

The comparison between point A and point B (Fig. 2) was used to examine the initial response to PHMB exposure. RNA yields from the cultures at these points were approximately $1 \mathrm{mg}$ (ml culture $)^{-1}$. Genes of known function 


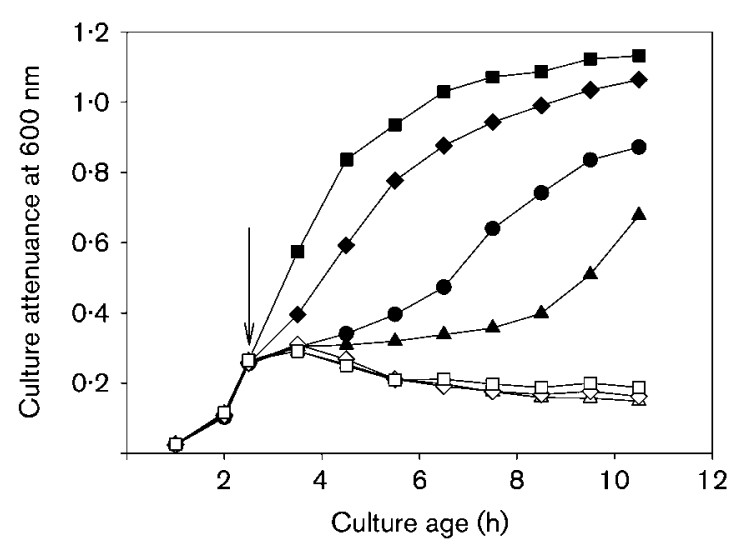

Fig. 1. Effect of addition of $\mathrm{PHMB}$ on growth of $E$. coli. PHMB was added at early exponential phase $(2.5 \mathrm{~h}$, as indicated by the arrow) to give final concentrations of: $\mathbf{\square}$, $0 \mathrm{mg} \mathrm{I}^{-1} ; \diamond, 2.5 \mathrm{mg} \mathrm{I}^{-1} ; \boldsymbol{\bullet}, 5 \mathrm{mg} \mathrm{I}^{-1} ; \boldsymbol{\Delta}, 7.5 \mathrm{mg} \mathrm{I}^{-1} ; \triangle$, $10 \mathrm{mg} \mathrm{I}^{-1} ; \diamond, 12.5 \mathrm{mg} \mathrm{I}^{-1} ; \square, 15 \mathrm{mg} \mathrm{I}^{-1}$.

with significantly altered expression profiles are listed in Table 3(a), classified according to the latest known functional assignments (http://genprotec.mbl.edu/ and Serres et al., 2004). Genes in known operons or possible operons are grouped together regardless of functional category. Genes of no known function (Table 3b) accounted for approximately half of those whose expression was altered immediately upon exposure to PHMB. The overwhelming majority of these were elevated in expression, and putative functions assigned to their protein products include transcriptional regulators and membrane proteins. Overall, upon

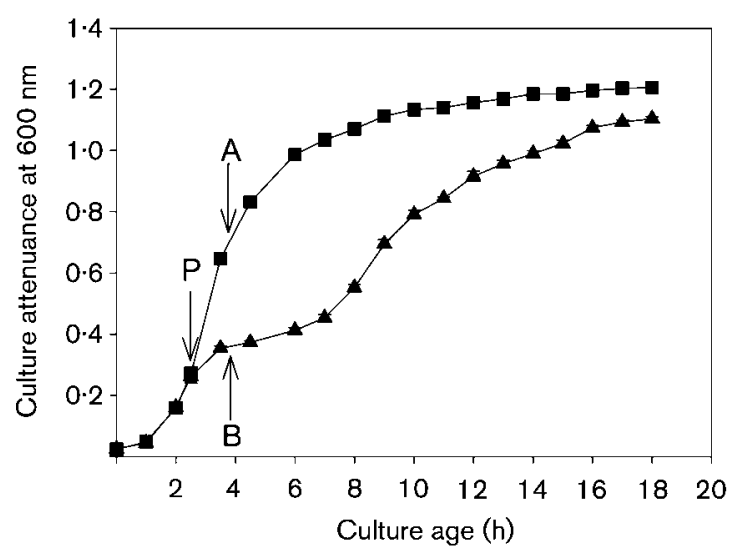

Fig. 2. Sampling for differential-expression profiling between PHMB-exposed ( $\boldsymbol{\Delta}$ ) and unexposed ( $\boldsymbol{\square})$ E. coli. PHMB (final concentration $7.5 \mathrm{mg} \mathrm{l}^{-1}$ ) was added to the test culture at point $P(2.5 \mathrm{~h})$. RNA samples were isolated from unexposed exponentially growing $E$. coli (point $A$ ) and from PHMB-exposed E. coli (point B) at the same culture age $(3.75 \mathrm{~h})$. It should be noted that error bars are included ( $2 \mathrm{SD}, n=3$ culture vessels); however, most errors are too small to see. exposure to PHMB, 71 genes were induced and 12 repressed significantly (Table 3).

\section{PHMB-phenotype of knockout mutants and overexpression strains}

Where possible, knockout strains were obtained for genes showing significant changes in transcript abundance and the deletion mutants tested for PHMB tolerance by determination of MIC. MICs were determined for the knockout strain itself (i.e. MG1655 $\Delta x \times x X$ ), an overexpression strain (i.e. MG1655 pMJA- $x \times x X$ ) and a complement of the knockout strain (i.e. MG1655 $\Delta x x x X$ pMJA- $x x x X$ ). The results of these MIC measurements are shown in Table 4.

\section{DISCUSSION}

\section{Outer membrane}

In the outer membrane PHMB was hitherto thought to interact primarily with LPS and to have a negligible interaction with proteins (Gilbert et al., 1990a). However, several genes associated with outer-membrane components (Oantigen, Rhs elements, flagella, fimbriae) were induced by exposure to PHMB, as now described.

Five genes that are either definitely $(r f a L, y e f I, r f c, r f b X$, Table 3a) or putatively ( $h t r L$, Table $3 \mathrm{~b}$ ) involved in the synthesis and processing of the $\mathrm{O}$-antigen, were induced immediately after exposure to PHMB. These five genes are part of the $r f b$ (O-antigen) gene cluster (b2032, b2035, b2037) and rfa (LPS core) gene cluster (b3622, b3618), respectively, and it is possible that other members of the clusters were induced, but missed in this analysis. Since $r f b X$ and $h t r L$ are involved in O-antigen biosynthesis and E. coli does not express a functional $\mathrm{O}$-antigen, the biological significance of these results remains unclear.

The genes encoding two lipid-anchored outer-membrane proteins, OsmB and VacJ, were also induced on exposure to PHMB (Table 3a). The osmB knockout strain was slightly more resistant to PHMB than the wild-type strain. However, complementation of $\operatorname{osmB}$ in this knockout strain, and overexpression in the wild-type, led to strong resistance (Table 4). Indeed, overexpression of $o s m B$ in the wild-type gave an MIC value more than twice that of the wild-type. OsmB is a multi-stress-responsive lipoprotein, being both osmotically inducible and regulated by RpoS to appear in stationary phase (Jung et al., 1990), and also under the control of a second independent regulatory system RcsCDB (Boulanger et al., 2005). This system is activated by environmental conditions that have the common consequence that they alter the envelope composition and/or topology, leading to the suggestion that perturbations in the cell envelope might be the inducing signal recognized by the RcsC sensor (Gottesman, 1995). Interestingly, cells express specific RcsCB-regulated genes in order to cope with the stress induced by chlorpromazine (Conter et al., 2002), which, like PHMB, is a cationic amphipathic molecule that 
Table 3. Genes of (a) known and (b) unknown function found to have significantly altered expression profiles in a standard analysis of PHMB amended compared with PHMB unamended E. coli at same culture age

\begin{tabular}{|c|c|c|c|}
\hline Gene & Blattner no & Gene product description & Fold change \\
\hline \multicolumn{4}{|c|}{ Nucleic acid associated } \\
\hline $\operatorname{rec} A$ & b2699 & DNA strand exchange and recombination protein & $+6 \cdot 6$ \\
\hline$d n a K$ & b0014 & Chaperone-heat-shock protein 70 & $+7 \cdot 4$ \\
\hline evgS & b2370 & Sensory histidine kinase regulating multidrug resistance & $+6 \cdot 9$ \\
\hline \multicolumn{4}{|c|}{ Translation } \\
\hline$r p l Y$ & b2185 & 50S Ribosomal protein L25 & $+9 \cdot 8$ \\
\hline hha & b0460 & Haemolysin expression modulator & $+16 \cdot 0$ \\
\hline rpsP & b2609 & 30S Ribosomal subunit protein S16 & $+14 \cdot 0$ \\
\hline tnaA & b3708 & Tryptophan deaminase & $-29 \cdot 3$ \\
\hline$t d c R$ & b3119 & $t d c A B C$ operon (threonine dehydratase) transcriptional activator & $+7 \cdot 1$ \\
\hline$c y s B$ & b1275 & $\begin{array}{l}\text { Transcriptional regulator of cysteine biosynthesis and regulator } \\
\text { of sulphur assimilation }\end{array}$ & $+8 \cdot 2$ \\
\hline$c b l$ & b1987 & Transcriptional regulator of cysteine biosynthesis & $+12 \cdot 2$ \\
\hline $\operatorname{asp} A$ & b4139 & Aspartate ammonia-lyase (aspartase) & $-21 \cdot 9$ \\
\hline \multicolumn{4}{|c|}{ Energy metabolism } \\
\hline$p f l B$ & b0903 & Pyruvate formate lyase I, induced anaerobically & $-18 \cdot 5$ \\
\hline$g l p D$ & b3426 & Glycerol-3-phosphate dehydrogenase (aerobic) & $+9 \cdot 6$ \\
\hline$f l g E$ & b1076 & Flagella hook protein FlgE & $-9 \cdot 8$ \\
\hline flgJ & b1081 & Flagella protein FlgJ & $-11 \cdot 3$ \\
\hline yadC & b0135 & Putative fimbrial-like protein & $+31 \cdot 9$ \\
\hline yehC & b2110 & Putative periplasmic fimbrial chaperone & $+7 \cdot 2$ \\
\hline \multicolumn{4}{|c|}{ Surface and outer-membrane associated } \\
\hline osmB & b1283 & Osmotically inducible lipoprotein B precursor & $+7 \cdot 4$ \\
\hline vacJ & b2346 & VacJ lipoprotein precursor & $+15 \cdot 3$ \\
\hline$r f a L$ & b3622 & O-antigen ligase & $+11 \cdot 6$ \\
\hline yefI & b2032 & Putative transferase & $+26 \cdot 0$ \\
\hline$r f c$ & b2035 & $\mathrm{O}$-antigen polymerase & $+5 \cdot 6$ \\
\hline$r f b X$ & b2037 & Putative $\mathrm{O}$-antigen transporter & $+16 \cdot 9$ \\
\hline \multicolumn{4}{|l|}{ Others } \\
\hline uspA & b3495 & Universal stress protein A & $-37 \cdot 2$ \\
\hline intB & b4271 & Prophage $\mathrm{P} 4$ integrase & $+5 \cdot 5$ \\
\hline $\operatorname{cpxP}$ & b3913 & Periplasmic repressor of $\mathrm{Cpx}$ regulon & $+17 \cdot 5$ \\
\hline b3914 & b3914 & Putative periplasmic protein & $+26 \cdot 1$ \\
\hline
\end{tabular}


Table 3. cont.

\begin{tabular}{|c|c|c|c|}
\hline Gene & Blattner no & Gene product description & Fold change \\
\hline \multicolumn{4}{|c|}{ Rhs associated } \\
\hline$b 0499$ & b0499 & Conserved protein & $+8 \cdot 9$ \\
\hline$y b b D$ & b0500 & Conserved hypothetical protein & $+13 \cdot 9$ \\
\hline$b 0501$ & b0501 & Unknown CDS & $+6 \cdot 6$ \\
\hline$y d c D$ & b1457 & Unknown CDS & $+14 \cdot 0$ \\
\hline$y b f D$ & b0706 & H-repeat associated protein & $+9 \cdot 3$ \\
\hline$r h s B$ & b3482 & RhsB core protein with unique extension & $+14 \cdot 3$ \\
\hline$y h h H$ & b3483 & Unknown CDS & $+19 \cdot 8$ \\
\hline yhiJ & b3488 & Conserved hypothetical protein & $+69 \cdot 4$ \\
\hline$h d e B$ & b3509 & Protein HDEB precursor & $-14 \cdot 8$ \\
\hline yeaC & b1777 & Conserved hypothetical protein & $-6 \cdot 4$ \\
\hline yaiN & b0357 & Conserved hypothetical protein & $+41 \cdot 0$ \\
\hline$y c g V$ & b1202 & Putative membrane protein & $+5 \cdot 2$ \\
\hline$y d j F$ & b1770 & Putative transcriptional regulator & $+8 \cdot 1$ \\
\hline$y g i G$ & b3046 & Putative outer-membrane usher protein & $+5 \cdot 5$ \\
\hline$b 0299$ & b0299 & Putative IS transposase & $+5 \cdot 9$ \\
\hline$y m g D$ & b1171 & Unknown CDS & $+15 \cdot 2$ \\
\hline$b 1172$ & b1172 & Conserved hypothetical protein & $+22 \cdot 5$ \\
\hline$y h i W$ & b3515 & Putative transcriptional regulator & $+6 \cdot 6$ \\
\hline$y h i X$ & b3516 & Putative transcriptional regulator & $+19 \cdot 0$ \\
\hline$h t r L$ & b3618 & Lipopolysaccharide biosynthesis & $+8 \cdot 6$ \\
\hline$y i i G$ & b3896 & Conserved protein & $+10 \cdot 0$ \\
\hline$y d j O$ & b1730 & Putative enzyme & $+6 \cdot 7$ \\
\hline$y f j W$ & b2642 & CP4-57 prophage & $+13 \cdot 9$ \\
\hline$b 1721$ & b1721 & Putative regulator & $+8 \cdot 4$ \\
\hline$y h a B$ & b3120 & Conserved protein & $+48 \cdot 8$ \\
\hline$b 2854$ & b2854 & Conserved protein, lysozyme like & $+19 \cdot 7$ \\
\hline$y j b M$ & b4048 & Conserved hypothetical protein & $+8 \cdot 4$ \\
\hline yebG & b1848 & $\begin{array}{l}\text { DNA damage inducible gene in SOS regulon, dependent on } \\
\text { cAMP, H-NS }\end{array}$ & $+13 \cdot 8$ \\
\hline$y j c F$ & b4066 & Conserved protein & $+10 \cdot 2$ \\
\hline$b 1527$ & b1527 & Conserved protein & $+6 \cdot 0$ \\
\hline yedM & b1935 & Unknown CDS & $+5 \cdot 5$ \\
\hline$y r h B$ & b3446 & Unknown CDS & $+6 \cdot 8$ \\
\hline$b 2863$ & b2863 & Unknown CDS & $+37 \cdot 5$ \\
\hline yeeN & b1983 & Conserved protein & $+9 \cdot 9$ \\
\hline b1963 & b1963 & Unknown CDS & $+13 \cdot 2$ \\
\hline
\end{tabular}

damages bacterial membranes (Silva et al., 1979). Thus the altered expression of osmB observed after exposure to PHMB is most easily attributed to cell envelope perturbation although, in the absence of data showing changes in either RpoS or RscCDB, the enforced entry into the stationary phase may also be a factor. 
Table 4. Phenotypic characterization of strains based upon MIC assay

The MIC of MG1655 was $3.5 \mathrm{mg} \mathrm{l}^{-1}$. The scale for the mean MIC in three replicate experiments is as follows: - - - - , MIC $\leqslant 1.25 \mathrm{mg} \mathrm{l}^{-1} ;----, 1.25 \mathrm{mg} \mathrm{l}^{-1}<\mathrm{MIC} \leqslant 1.75 \mathrm{mg} \mathrm{l}^{-1} ; \quad---, 1.75 \mathrm{mg} \mathrm{l}^{-1}$

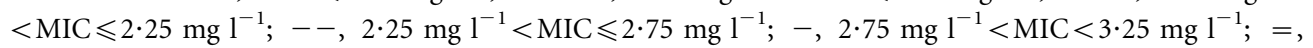
$3.25 \mathrm{mg} \mathrm{l}^{-1} \leqslant \mathrm{MIC} \leqslant 3.75 \mathrm{mg} \mathrm{l}^{-1} ; \quad+, \quad 3.75 \mathrm{mg} \mathrm{l}^{-1}<\mathrm{MIC}<4.50 \mathrm{mg} \mathrm{l}^{-1} ; \quad++, \quad 4.50 \mathrm{mg} \mathrm{l}^{-1} \leqslant \mathrm{MIC}<$ $5 \cdot 25 \mathrm{mg} \mathrm{l}^{-1} ;+++, 5 \cdot 25 \mathrm{mg} \mathrm{l}^{-1} \leqslant \mathrm{MIC}<6.75 \mathrm{mg} \mathrm{l}^{-1} ;++++, 6 \cdot 75 \mathrm{mg} \mathrm{l}^{-1} \leqslant$ MIC. Knockout strains that were not available for analysis are indicated by NA.

\begin{tabular}{|c|c|c|c|}
\hline \multirow[t]{2}{*}{ Gene } & \multicolumn{3}{|c|}{ MIC for strain in which indicated gene was: } \\
\hline & Knocked out & Knockout complemented & Overexpressed \\
\hline \multicolumn{4}{|c|}{ DNA/RNA-associated } \\
\hline hns & --- & -- & + \\
\hline $\operatorname{stp} A$ & NA & NA & --- \\
\hline $\operatorname{rec} A$ & - & + & ++++ \\
\hline yebG & $=$ & + & +++ \\
\hline \multicolumn{4}{|c|}{ Metabolism } \\
\hline $\operatorname{tna} A$ & $\mathrm{NA}$ & $\mathrm{NA}$ & $=$ \\
\hline \multicolumn{4}{|c|}{ Periplasm-associated } \\
\hline $\operatorname{cpxP}$ & - & - & -- \\
\hline hdeA & -- & --- & ++ \\
\hline$h d e B$ & NA & NA & ++ \\
\hline \multicolumn{4}{|c|}{ Outer membrane } \\
\hline osmB & + & ++++ & +++++ \\
\hline \multicolumn{4}{|c|}{ Flagella and fimbriae } \\
\hline flgJ & + & ---- & $=$ \\
\hline$f l g E$ & NA & NA & $=$ \\
\hline \multicolumn{4}{|c|}{ Rhs elements } \\
\hline$r h s B$ & NA & NA & +++ \\
\hline$r h s D$ & NA & NA & ++ \\
\hline$r h s E$ & - & --- & -- \\
\hline \multicolumn{4}{|c|}{ Unknown function } \\
\hline yaiN & NA & NA & $=$ \\
\hline$y c g W$ & NA & NA & --- \\
\hline yhaB & NA & NA & $=$ \\
\hline
\end{tabular}

\section{Rhs elements}

Exposure to PHMB caused rapid and strong induction of $r h s D(40 \cdot 0)$, rhsE $(16 \cdot 1)$ and $r h s B(14 \cdot 3)$ together with a number of associated genes downstream from each of these (Table 3). Of the remaining two Rhs elements, expression of $r h s A$ was unaffected and $r h s C$ was significantly upregulated in two of the three replicate experiments (data not shown). However, PHMB also induced additional genes yibJ (b3595) and $y b f D$ (b0706) located downstream from rhsA (b3593) and $r h s C$ (b0700), respectively (Table 3 ), perhaps suggesting that all five elements were induced in response to the presence of PHMB. Because these rhsABCDE genes have similar sequences, cross-hybridization artefacts might account for some of the observed signals. However, because PHMB also induced the associated downstream genes that show little or no sequence similarity to each other or the rhs genes themselves, it is likely that the signals for the latter are true positives.

The function of the Rhs elements (first identified as being recombinational hot spots) has remained elusive since their discovery over 20 years ago (Lin et al., 1984). They are not essential but are conserved among E. coli strains. Hitherto, attempts to express them under laboratory conditions have failed, so the discovery of induction by exposure to PHMB is both remarkable and a potentially important step in the elucidation of gene function. The rhs core ORF $(\sim 3 \cdot 71 \mathrm{~kb})$ contains a peptide motif $x x G x x R Y x Y D x x G R L(I$ or $T) x x x x$ that is repeated 28 times. Hill et al. (1994) suggested that these are cell-surface ligand-binding proteins, based on similarity with the Bacillus WapA (wall-associated protein) gene sequence (Foster, 1993), and that the downstream ORFs associated with the rhs core ORFs may play roles in the transport and processing of the core ORFs (which lack obvious signal sequences).

Overexpression of the core $r h s B$ or $r h s D$ in the wild-type led to increased PHMB resistance (Table 4), consistent with their strong induction in the wild-type on exposure to $\mathrm{PHMB}$ and possibly indicative of a protective role. Overexpression of $r h s A$ or $r h s C$ caused no change in PHMB 
sensitivity (data not shown), consistent with our observation that PHMB failed to induce these genes. Overexpression of rhsE was anomalous in producing little change, despite its strong induction by PHMB. However, absence of a strong phenotype in the overexpressing strain is consistent with the notion that rhsE is the only Rhs element that is 'non-functional' in E. coli K-12 (Sadosky et al., 1991; Wang et al., 1998). The absence of a clear pattern of PHMB resistance among strains overexpressing rhs genes and downstream ORFs confounds unequivocal interpretation of their role in PHMB resistance. Nevertheless, induction of these large putative cell-surface proteins could provide a physical barrier preventing PHMB from interacting directly with LPS in the outer membrane. Alternatively, these anionic proteins [theoretical pI 6.21, with excess of Glu and Asp residues (totalling 185) over Arg and Lys (totalling 139)] may possibly be released into the growth medium, perhaps sequestering the cationic PHMB, and indeed it has been suggested that the homologous WapA may actually be clipped from the membrane and secreted (Foster, 1993). Based on their possible cell-surface location (Hill et al., 1994), these Rhs proteins could also play a role in cellular aggregation, thus reducing $\mathrm{PHMB}$ exposure by reducing the area of exposed cell surface (see below).

\section{Flagella, fimbriae and pili}

Exposure to PHMB caused induction of hypothetical pilusassembly genes $y a d C$ and $y e h C$ (Table $3 \mathrm{a}$ ), a putative adhesion gene $y c g V$ and, downstream, a putative GTP-binding pilus chaperone $y c h F$ (Table $3 \mathrm{~b}$ ). At the same time, the flagella-associated genes $f l g E$ and $f l g J$ were down-regulated. The latter are members of a flagella-associated operon that includes $f l g B C D E F G H I J K L$. It is likely that all of the operon has had a decrease in expression after exposure to PHMB, but that only flgE and flgJ have been identified as being significantly altered. Reasons for this could include variation in transcript stability, primer efficiency in the reverse transcription step and hybridization. Pili are known to be used as adherence factors (Low et al., 1987) and to link cells to form aggregates (when they become fimbriae). Thus the switch in production from flagella to pili is consistent with cell aggregation as a response to PHMB exposure. Cellular self-association mediated by pili might protect the innermost cells from PHMB until further changes in gene expression allow cells to recover and grow in the presence of PHMB. The observed rapid decrease in culture attenuance after challenge with higher concentrations of PHMB (Fig. 1) is consistent with cell-cell aggregation.

A flgJ knockout strain was slightly more resistant to PHMB in comparison with the wild-type strain. Overexpression of $f l g J$ in this knockout (but not in the wild-type) strain led to PHMB sensitivity. FlgJ is bifunctional, containing a Cterminal muramidase to degrade peptidoglycan at the site of flagellum formation (Nambu et al., 1999) and a putative flagellar motor rod-capping role (Hirano et al., 2001). FlgJ, through its muramidase activity, is responsible for the hydrolysis of the peptidoglycan in the periplasm, creating space for the rod assembly to penetrate. Presumably, overexpression of $f l g J$ would increase the rate of breakdown of peptidoglycan in the periplasm, thus allowing PHMB easier access to the cytoplasmic membrane. The resistance of the flgJ knockout strain could therefore arise from diminished breakdown of peptidoglycan.

\section{Periplasm}

Although PHMB needs to traverse the periplasmic space en route to the cytoplasmic membrane, relatively few periplasmic-protein-encoding genes were changed in response to the presence of PHMB. Exposure to PHMB induced the periplasmic repressor of the $\mathrm{Cpx}$ regulon $(\mathrm{CpxP})$ approximately 20 -fold and, in keeping with its repressor function, no other members appeared to be induced. However the $c p x$ system is complex and $c_{p} x P$ itself serves more than one function. The system is activated by misfolded periplasmic proteins and controls not only a stress response but also the assembly of pili. CpxP is a feedback repressor of the $c p x$ pathway, dependent for its activity on the presence of the two-component sensor-kinase, CpxA. The ability to autoactivate and then subsequently repress the $c p x$ pathway is believed (Raivio et al., 1999) to allow for a temporary amplification of the Cpx response that may be important in rescuing cells from transitory stresses, as is the present case with sublethal exposure to PHMB. CpxP is also a periplasmic chaperone (DiGiuseppe \& Silhavy, 2003; Duguay \& Silhavy, 2004) known to bind to misfolded pilus subunits (Hung et al., 2001) and it could be induced directly in response to damage to pili caused by PHMB.

The $h d e B$ gene in the $h d e A B$ operon was down-regulated around 15-fold immediately after exposure to PHMB (hdeA was down-regulated approximately eightfold initially, but only significantly in two of three experiments). These genes are linked to acid resistance and may act as chaperones by preventing the aggregation of denatured proteins and thus may also act in a similar fashion in response to the chaotropic action of PHMB.

The alterations in expression of genes encoding periplasmic proteins would be expected to be much greater if extensive damage to the contents of the periplasm was occurring. This relative stability in gene expression therefore suggests that the route to the cytoplasmic membrane by PHMB is direct and that it has very little interaction with the contents of the periplasm.

\section{Cytoplasmic membrane}

Many genes involved in transport/binding functions of the cytoplasmic membrane were altered in response to PHMB, in particular those involved in sugar transport. Exposure to PHMB caused $r b s D$ to become down-regulated immediately 12 -fold. RbsD is involved in the high-affinity transport of ribose. The gat operon, involved in the galactitol-specific phosphotransferase system, was also down-regulated on PHMB exposure. In experiments over a longer time frame in 
which comparison of unexposed with exposed-recovered cells was made (data not shown), lldP (encoding a lactate permease) was down-regulated approximately sixfold. Shutdown of transport systems when challenged with a toxic substance seems a logical strategy but phenotypic analysis of the few available knockout strains gave no coherent set of results although the tolerance to $\mathrm{PHMB}$ was altered in some strains (data not shown).

\section{Central metabolism}

Tryptophan deaminase (tnaA) and an associated leader peptide (tnaL) were down-regulated approximately 29 - and 34 -fold, respectively (Table 2). Besides an apparent role in alkaline stress (Blankenhorn et al., 1999; Bordi et al., 2003; Stancik et al., 2002; Yohannes et al., 2004), TnaA converts tryptophan to indole, which is believed to act as a signal molecule (Wang et al., 2001) in the onset of stationary phase (Lacour \& Landini, 2004) and as a stimulator of biofilm production (Di Martino et al., 2002, 2003). Thus, although PHMB exposure produced an enforced stationary phase, cells did not appear to use the tnaAL pathway to signal the need for biofilm formation.

The aspartase gene (aspA) was down-regulated 22-fold (Table 2) by PHMB exposure, and the corresponding knockout strain was much more resistant than the wildtype (Table 4). Complementing the knockout with the aspA expression plasmid restored some sensitivity, and overexpression in the wild-type produced hypersensitivity to PHMB. The aspA gene encodes an aspartate ammonia lyase (aspartase) that converts L-aspartate into fumarate and ammonia. The enzyme is repressed when aspartate is needed for other biosynthetic purposes (Kim et al., 2004), but under anaerobic conditions the enzyme is induced in order to generate fumarate as an electron acceptor (Bronder et al., 1982). However, the enzyme is also produced under aerobic conditions, suggesting it has multiple roles (Golby et al., 1998). Repression of aspA gene expression has also been noted as an initial response in other stressful conditions (e.g. Brocklehurst \& Morby, 2000; Polen et al., 2003) and indeed AspA appears to interact with a conserved histidinephosphotransferase domain of a two-component signalling system (Salinas \& Contreras, 2003), suggesting involvement in transcriptional regulation. Nevertheless, although expression of the aspA gene significantly degraded resistance to $\mathrm{PHMB}$, an adequate explanation remains to be found.

Expression of two genes that affect formate metabolism was altered by exposure to PHMB. The frmR (formerly yaiN) gene product, which is a repressor of frm $A B$ that encodes enzymes for oxidation of formaldehyde to formate (Herring \& Blattner, 2004), was markedly induced (41-fold) by PHMB. In addition, pyruvate-formate lyase $(p f l B)$ was repressed (Table 3a). Thus PHMB exposure suppressed formation of formate, which is regarded as a signature molecule in the fermenting E. coli cell (Sawers, 2005). Formate probably regulates the hyf operon encoding a respiration-linked proton-translocating formate hydrogen lyase that generates dihydrogen from formate under anaerobic conditions (Andrews et al., 1997). These results, together with the strong suppression of aspartase, suggest that part of the response to PHMB is to deny the cells any opportunity to switch to anaerobic metabolism.

It is possible that the repression of tnaA and aspA serves as an indirect response to preserve amino acids needed to replace proteins damaged by exposure to PHMB. This is consistent with the observed induction of the transcriptional master regulator of sulphur assimilation into cysteine, $c y s B$, and its associated accessory element $c b l$ (induced approximately 8 - and 12-fold, respectively).

\section{DNA-binding proteins}

PHMB altered the expression of several genes encoding DNA-binding proteins, other than specific transcriptional regulators. For example, transcripts for $\operatorname{rec} A$, xseA (an exodeoxyribonuclease) and $\operatorname{stp} A$ [a $\mathrm{H}-\mathrm{NS}$ homologue and potential H-NS chaperone (Dorman \& Deighan, 2003)] were induced approximately seven-, seven- and ninefold, respectively, immediately after exposure to PHMB. Overexpression of $\operatorname{stp} A$ (encoding a chaperone and homologue of H-NS, the histone-like nucleoid structuring protein) increased the sensitivity to PHMB; a knockout strain was unavailable for assay. Given the strong sequence similarity between StpA and H-NS but their distinct expression patterns (Dorman et al., 1999), we also assessed the PHMB tolerance of strains expressing hns variably. Interestingly, resistance to PHMB was lost in an hns knockout strain, partially regained in the complemented knockout, and enhanced (compared to the wild-type) in the overexpressing strain. Thus PHMB tolerance in E. coli was directly correlated with the level of H-NS expression. Because H-NS globally represses many unrelated genes (Lammi et al., 1984), the apparent sensitivity in the hns knockout strain could be caused by a deregulation of H-NS-regulated genes, but it could also be caused by a change in DNA structure. H-NS regulates by binding to at least two patches of curved DNA to create loops in which RNA polymerase is trapped (Dorman, 2004; Dorman \& Deighan, 2003). Moreover, the smallest functional units are dimers which may oligomerize and effectively cross-link adjacent DNA segments of DNA through an oligomeric matrix of H-NS (Dorman, 2004). This process could have a direct protective role against PHMB, because the latter is also known to interact strongly and cooperatively with DNA to create cross-links (Allen et al., 2004) and thus potentially to interfere with DNAmetabolism (replication, repair, transcription, etc.). Competitive binding by $\mathrm{H}-\mathrm{NS}$ would mitigate against such adverse effects of PHMB.

The regulatory networks in E. coli are highly integrated and complex, and rarely, if ever, rely on a single regulator in response to stress. Indeed, Table 3 shows that several other known or putative transcriptional regulators (e.g. hha, $y b c M, t d c R, c y s B, c b l)$ are induced by PHMB and their binding to DNA may also contribute to a DNA-shielding 
effect. That stpA and hns give opposing PHMB tolerance phenotypes when expressed in wild-type E. coli provides additional evidence for the difference in biological role of the $\operatorname{stp} A$ and $h n s$ gene products.

The recA knockout was sensitive to PHMB and complementation of recA in this knockout strain led to modest PHMB resistance. Overexpression of $r e c A$ in the wild-type strain led to a stronger resistance to PHMB. If PHMB is indeed gaining access to the cytoplasm and interacting with genomic DNA (Allen et al., 2004), the induction of RecA could either help to repair the damage effected by PHMB, or be part of a damage signal that leads to other events affecting the phenotype, or contribute to an increased capacity to generate diversity (cf. $y c g W$ below).

Expression of $y e b G$ (Table $3 \mathrm{~b}$ ) is a part of the SOS response to damaged DNA and part of the entry into stationary phase (Lomba et al., 1997; Oh \& Kim, 1999). A yebG knockout strain showed no difference to the wild-type in sensitivity to PHMB but the complemented knockout strain became slightly more resistant and overexpression in the wild-type strain produced strong resistance. Induction of yebG by PHMB may therefore be a response either to DNA damage or the entry to an enforced stationary (bacteriostatic) phase. Although the transcriptional control of $y e b G$ is partly characterized (it is dependent on H-NS and cAMP, but does not require RpoS), no biological function has been found, to date, for YebG (Oh \& Kim, 1999).

\section{Potential mutator gene}

The $y c g W$ gene, which was strongly induced by PHMB (Table 3), was previously isolated in a screen for mutator function (Yang et al., 2004). Mutator cells can have increased fitness, and are selected during exposure to fluctuating environments (see Wright, 2004, for review). Conversely, the overexpression of a mutator (i.e. from a high-copy plasmid) would probably be detrimental to the cell if it conferred too great a mutational load, and this may explain the lesser tolerance seen to PHMB in $y c g W$ expressing cells (Table 4).

\section{The global response of E. coli to PHMB}

In terms of cellular location, E. coli responded to bacteriostatic levels of PHMB by altering the expression of many genes functioning at all levels of cell ultrastructure, i.e. the outer membrane, periplasm, inner membrane and cytoplasmic domains. On the other hand, some of the transcriptional changes were clustered around relatively few specific aspects of cell physiology. First, genes associated with stresses including acid resistance, alkali resistance, osmotic shock and cell-envelope perturbation were altered in expression alongside the controlling factors of the Evg and Cpx response systems. These systems are all involved with sensing and responding to environmental insults that affect the outer membrane, periplasm and cytoplasmic membrane. Since PHMB interacts initially with this cell envelope, the induction of members of these systems is not surprising. Second, several transcriptional changes (loss of flagella, pilus formation, $c p x$-mediated repair to pili) point towards cell-cell aggregation as a strategy to minimize exposure of cell surface to PHMB. This hypothesis is in keeping with observations that E. coli MG1655 cells, when viewed by light microscopy, both aggregate and elongate on exposure to PHMB at the same concentrations as used in the transcript profiling above (data not shown). The third and most novel and intriguing feature of transcript profiles was the induction of the $\sigma^{\mathrm{H}}$ (heat shock) response, SOS (DNA damage) response and other DNA metabolism-associated genes, which implied that significant damage was occurring to DNA in the cytoplasm. Although this would be almost predictable at bactericidal levels of PHMB (when severe disruption of the inner membrane is thought to occur), these experiments were performed at bacteriostatic levels of PHMB from which the cells, shortly after, recover. These findings led us to expand the theory for the PHMB mechanism of action, as discussed below.

Despite these rationalizations in terms of stress response, aggregation, and DNA-damage limitation, much of the E. coli response could not be interpreted as clearly. For example, lipopolysaccharide is thought to be an important exclusion barrier against PHMB, but relatively few genes associated with LPS metabolism were transcriptionally altered. Those that were affected are mostly involved in the synthesis of the O-antigen (a polysaccharide attached to the lipid core of a LPS). However, the O-antigen is not thought to be functional in K-12 strains of E. coli. Furthermore, the physiological reasons behind the strong induction of the Rhs elements still remain to be elucidated. Since their discovery, the function of the Rhs elements has remained elusive, particularly because no conditions have previously been found that lead to their induction.

\section{A new theory for the PHMB mechanism of action}

Recently we demonstrated a strong cooperative binding between PHMB and DNA, leading to co-precipitation in vitro (Allen et al., 2004). This discovery, taken together with the present results, indicates that the critical factor underlying the different effects at bacteriostatic and bactericidal levels of PHMB may not be the ability to disrupt the inner membrane (as previously thought), but the direct interaction between PHMB and genomic DNA. At lower (bacteriostatic) concentrations, the damage caused by the interaction between PHMB and DNA could be tolerable and even repairable. Since binding is highly cooperative, small increases in PHMB concentrations can cause a massive increase in perturbation of DNA function and/or precipitation, thus leading to cell death. This could account for the dose dependence observed by Broxton et al. (1983) and Davies et al. (1968) and the aggregation of phosphorus near the cell wall in PHMB-treated Acanthamoeba (Khunkitti et al., 1998), and could help explain the rapid switch, over a small concentration range, between bacteriostatic and bactericidal levels seen in this study (Fig. 1). This theory 
also underpins the central feature that allows widespread use of PHMB as a disinfectant, namely that it has very low mammalian toxicity and that eukaryotes tested tend to have higher MICs than prokaryotes. In eukaryotic cells, the much more extensive compartmentation of genomic DNA and the presence of internal structures are further barriers blocking the direct interaction between PHMB and DNA.

\section{ACKNOWLEDGEMENTS}

We would like to thank Dr Simon Andrews for supplying the E. coli ORF overexpression plasmids, Cardiff University for financial support and Avecia for supply of specialty chemicals

\section{REFERENCES}

Allen, M. J., Morby, A. P. \& White, G. F. (2004). Cooperativity in the binding of the cationic biocide polyhexamethylene biguanide to nucleic acids. Biochem Biophys Res Commun 318, 397-404.

Andrews, S. C., Berks, B. C., McClay, J., Ambler, A., Quail, M. A., Golby, P. \& Guest, J. R. (1997). A 12-cistron Escherichia coli operon (hyf) encoding a putative proton-translocating formate hydrogenlyase system. Microbiology 143, 3633-3647.

Blankenhorn, D., Phillips, J. \& Slonczewski, J. L. (1999). Acid- and base-induced proteins during aerobic and anaerobic growth of Escherichia coli revealed by two-dimensional gel electrophoresis. J Bacteriol 181, 2209-2216.

Blattner, F. R., Plunkett, G. R., Bloch, C. A. \& 14 other authors (1997). The complete genome sequence of Escherichia coli K-12. Science 277, 1453-1474.

Bordi, C., Theraulaz, L., Mejean, V. \& Jourlin-Castelli, C. (2003). Anticipating an alkaline stress through the Tor phosphorelay system in Escherichia coli. Mol Microbiol 48, 211-223.

Boulanger, A., Francez-Charlot, A., Conter, A., Castanie-Cornet, M. P., Cam, K. \& Gutierrez, C. (2005). Multistress regulation in Escherichia coli: expression of osmB involves two independent promoters responding either to sigmaS or to the RcsCDB His-Asp phosphorelay. J Bacteriol 187, 3282-3286.

Brocklehurst, K. R. \& Morby, A. P. (2000). Metal-ion tolerance in Escherichia coli: analysis of transcriptional profiles by gene-array technology. Microbiology 146, 2277-2282.

Bronder, M., Mell, H., Stupperich, E. \& Kroger, A. (1982). Biosynthetic pathways of Vibrio succinogenes growing with fumarate as terminal electron acceptor and sole carbon source. Arch Microbiol 131, 216-223.

Broxton, P., Woodcock, P. M. \& Gilbert, P. (1983). A study of the antibacterial activity of some polyhexamethylene biguanides towards Escherichia coli ATCC 8739. J Appl Bacteriol 54, 345-353.

Broxton, P., Woodcock, P. M. \& Gilbert, P. (1984). Interaction of some polyhexamethylene biguanides and membrane phospholipids in Escherichia coli. J Appl Bacteriol 57, 115-124.

Cazzaniga, A., Serralta, V., Davis, S., Orr, R., Eaglstein, W. \& Mertz, P. M. (2002). The effect of an antimicrobial gauze dressing impregnated with $0 \cdot 2$-percent polyhexamethylene biguanide as a barrier to prevent Pseudomonas aeruginosa wound invasion. Wounds-Compend Clin Res Pract 14, 169-176.

Cohen, S. S. (1998). A Guide To Polyamines, pp. 185-230. Oxford: Oxford University Press.

Conter, A., Sturny, R., Gutierrez, C. \& Cam, K. (2002). The RcsCB HisAsp phosphorelay system is essential to overcome chlorpromazineinduced stress in Escherichia coli. J Bacteriol 184, 2850-2853.
Cox, N. A., Bailey, J. S. \& Berrang, M. E. (1998). Bactericidal treatment of hatching eggs. I. Chemical immersion treatments and salmonella. J Appl Poult Res 7, 347-350.

Cox, N. A., Berrang, M. E., Buhr, R. J. \& Bailey, J. S. (1999). Bactericidal treatment of hatching eggs. II. Use of chemical disinfectants with vacuum to reduce salmonella. J Appl Poult Res 8, 321-326.

Datsenko, K. A. \& Wanner, B. L. (2000). One-step inactivation of chromosomal genes in Escherichia coli K-12 using PCR products. Proc Natl Acad Sci U S A 97, 6640-6645.

Davies, A., Bentley, M. \& Field, B. S. (1968). Comparison of the action of vantocil, cetrimide and chlorhexidine on Escherichia coli and its spheroplasts and the protoplasts of gram positive bacteria. $J$ Appl Bacteriol 31, 448-461.

DiGiuseppe, P. A. \& Silhavy, T. J. (2003). Signal detection and target gene induction by the CpxRA two-component system. J Bacteriol 185, 2432-2440.

Di Martino, P., Merieau, A., Phillips, R., Orange, N. \& Hulen, C. (2002). Isolation of an Escherichia coli strain mutant unable to form biofilm on polystyrene and to adhere to human pneumocyte cells: involvement of tryptophanase. Can J Microbiol 48, 132-137.

Di Martino, P., Fursy, R., Bret, L., Sundararaju, B. \& Phillips, R. S. (2003). Indole can act as an extracellular signal to regulate biofilm formation of Escherichia coli and other indole-producing bacteria. Can J Microbiol 49, 443-449.

Donoso, R., Mura, J. J. \& Lopez, M. (2002). Acanthamoeba keratitis treated with propamidine and polyhexamethyl biguanide (PHMB). Rev Med Chil 130, 396-401.

Dorman, C. J. (2004). H-NS: a universal regulator for a dynamic genome. Nat Rev Microbiol 2, 391-400.

Dorman, C. J. \& Deighan, P. (2003). Regulation of gene expression by histone-like proteins in bacteria. Curr Opin Genet Dev 13, 179-184.

Dorman, C. J., Hinton, J. C. \& Free, A. (1999). Domain organization and oligomerization among H-NS-like nucleoid-associated proteins in bacteria. Trends Microbiol 7, 124-128.

Duguay, A. R. \& Silhavy, T. J. (2004). Quality control in the bacterial periplasm. Biochim Biophys Acta 1694, 121-134.

Foster, S. J. (1993). Molecular analysis of three major wall-associated proteins of Bacillus subtilis 168: evidence for processing of the product of a gene encoding a $258 \mathrm{kDa}$ precursor two-domain ligandbinding protein. Mol Microbiol 8, 299-310.

Gerdes, S. Y., Scholle, M. D., Campbell, J. W. \& 18 other authors (2003). Experimental determination and system level analysis of essential genes in Escherichia coli MG1655. J Bacteriol 185, 5673-5684.

Gilbert, P., Pemberton, D. \& Wilkinson, D. E. (1990a). Barrier properties of the Gram-negative cell envelope towards high molecular weight polyhexamethylene biguanides. J Appl Bacteriol 69, 585-592.

Gilbert, P., Pemberton, D. \& Wilkinson, D. E. (1990b). Synergism within polyhexamethylene biguanide biocide formulations. $J$ Appl Bacteriol 69, 593-598.

Golby, P., Kelly, D. J., Guest, J. R. \& Andrews, S. C. (1998). Transcriptional regulation and organization of the $d c u A$ and $d c u B$ genes, encoding homologous anaerobic C4-dicarboxylate transporters in Escherichia coli. J Bacteriol 180, 6586-6596.

Gottesman, S. (1995). Regulation of capsule synthesis: modification of the two-component paradigm by an accessory unstable regulator. In Two-Component Signal Transduction, pp. 253-262. Edited by J. A. Hoch \& T. J. Silhavy. Washington, DC: American Society for Microbiology.

Gray, T. B., Gross, K. A., Cursons, R. T. M. \& Shewan, J. F. (1994). Acanthamoeba-keratitis - a sobering case and a promising new treatment. Aust N Z J Ophthalmol 22, 73-76. 
Herring, C. D. \& Blattner, F. R. (2004). Global transcriptional effects of a suppressor tRNA and the inactivation of the regulator frmR. $J$ Bacteriol 186, 6714-6720.

Hill, C. W., Sandt, C. H. \& Vlazny, D. A. (1994). Rhs elements of Escherichia coli - a family of genetic composites each encoding a large mosaic protein. Mol Microbiol 12, 865-871.

Hirano, T., Minamino, T. \& Macnab, R. M. (2001). The role in flagellar rod assembly of the N-terminal domain of Salmonella FlgJ, a flagellum-specific muramidase. J Mol Biol 312, 359-369.

Hiti, K., Walochnik, J., Haller-Schober, E. M., Faschinger, C. \& Aspock, H. (2002). Viability of Acanthamoeba after exposure to a multipurpose disinfecting contact lens solution and two hydrogen peroxide systems. Br J Ophthalmol 86, 144-146.

Hung, D. L., Raivio, T. L., Jones, C. H., Silhavy, T. J. \& Hultgren, S. J. (2001). Cpx signaling pathway monitors biogenesis and affects assembly and expression of $\mathrm{P}$ pili. EMBO $J$ 20, 1508-1518.

Ikeda, T., Tazuke, S., Bamford, C. H. \& Ledwith, A. (1984). Interaction of polymeric biguanide biocide with phospholipid membranes. Biochim Biophys Acta 54, 796-799.

Jung, J. U., Gutierrez, C., Martin, F., Ardourel, M. \& Villarejo, M. (1990). Transcription of $o s m B$, a gene encoding an Escherichia coli lipoprotein, is regulated by dual signals. Osmotic stress and stationary phase. J Biol Chem 265, 10574-10581.

Khunkitti, W., Hann, A. C., Lloyd, D., Furr, J. R. \& Russell, A. D. (1998). Biguanide-induced changes in Acanthamoeba castellanii: an electron microscopic study. J Appl Microbiol 84, 53-62.

Khunkitti, W., Hann, A. C., Lloyd, D., Furr, J. R. \& Russell, A. D. (1999). X-ray microanalysis of chlorine and phosphorus content in biguanide-treated Acanthamoeba castellanii. J Appl Microbiol 86, 453-459.

Kim, Y. H., Park, J. S., Cho, J. Y., Cho, K. M., Park, Y. H. \& Lee, J. (2004). Proteomic response analysis of a threonine-overproducing mutant of Escherichia coli. Biochem J 381, 823-829.

Kusnetsov, J. M., Tulkki, A. I., Ahonen, H. E. \& Martikainen, P. J. (1997). Efficacy of three prevention strategies against legionella in cooling water systems. J Appl Microbiol 82, 763-768.

Lacour, S. \& Landini, P. (2004). SigmaS-dependent gene expression at the onset of stationary phase in Escherichia coli: function of sigmaS-dependent genes and identification of their promoter sequences. J Bacteriol 186, 7186-7195.

Lammi, M., Paci, M., Pon, C. L., Losso, M. A., Miano, A., Pawlik, R. T., Gianfranceschi, G. L. \& Gualerzi, C. O. (1984). Proteins from the prokaryotic nucleoid: biochemical and ${ }^{1} \mathrm{H}$ NMR studies on three bacterial histone-like proteins. Adv Exp Med Biol 179, 467-477.

Lin, R. J., Capage, M. \& Hill, C. W. (1984). A repetitive DNA sequence, rhs, responsible for duplications within the Escherichia coli K-12 chromosome. J Mol Biol 177, 1-18.

Lomba, M. R., Vasconcelos, A. T., Pacheco, A. B. \& de Almeida, D. F. (1997). Identification of $y e b G$ as a DNA damage-inducible Escherichia coli gene. FEMS Microbiol Lett 156, 119-122.

Low, D., Braaten, B. \& Woude, V. D. (1987). Fimbriae. In Escherichia coli and Salmonella typhimurium: Cellular and Molecular Biology. Edited by F. C. Neidhardt and others. Washington, DC: American Society for Microbiology.

Messick, C. R., Pendland, S. L., Moshirfar, M., Fiscella, R. G., Losnedahl, K. J., Schriever, C. A. \& Schreckenberger, P. C. (1999). In-vitro activity of polyhexamethylene biguanide (PHMB) against fungal isolates associated with infective keratitis. $J$ Antimicrob Chemother 44, 297-298.

Mori, H., Isono, K., Horiuchi, T. \& Miki, T. (2000). Functional genomics of Escherichia coli in Japan. Res Microbiol 151, 121-128.
Nambu, T., Minamino, T., Macnab, R. M. \& Kutsukake, K. (1999). Peptidoglycan-hydrolyzing activity of the FlgJ protein, essential for flagellar rod formation in Salmonella typhimurium. J Bacteriol 181, 1555-1561.

Narasimhan, S., Madhavan, H. N. \& Therese, L. K. (2002). Development and application of an in vitro susceptibility test for Acanthamoeba species isolated from keratitis to polyhexamethylene biguanide and chlorhexidine. Cornea 21, 203-205.

Oh, T. J. \& Kim, I. G. (1999). Identification of genetic factors altering the SOS induction of DNA damage-inducible yebG gene in Escherichia coli. FEMS Microbiol Lett 177, 271-277.

Payne, J. D. \& Kudner, D. W. (1996). A durable antiodor finish for cotton textiles. Text Chem Color 28, 28-30.

Polen, T., Rittmann, D., Wendisch, V. F. \& Sahm, H. (2003). DNA microarray analyses of the long-term adaptive response of Escherichia coli to acetate and propionate. Appl Environ Microbiol 69, 1759-1774.

Raivio, T. L., Popkin, D. L. \& Silhavy, T. J. (1999). The Cpx envelope stress response is controlled by amplification and feedback inhibition. J Bacteriol 181, 5263-5272.

Rosin, M., Welk, A., Bernhardt, O., Ruhnau, M., Pitten, F. A., Kocher, T. \& Kramer, A. (2001). Effect of a polyhexamethylene biguanide mouthrinse on bacterial counts and plaque. $J$ Clin Periodontol 28, 1121-1126.

Rosin, M., Welk, A., Kocher, T., Majic-Todt, A., Kramer, A. \& Pitten, F. A. (2002). The effect of a polyhexamethylene biguanide mouthrinse compared to an essential oil rinse and a chlorhexidine rinse on bacterial counts and 4-day plaque regrowth. J Clin Periodontol 29, 392-399.

Sadosky, A. B., Gray, J. A. \& Hill, C. W. (1991). The Rhsd-E subfamily of Escherichia coli K-12. Nucleic Acids Res 19, 7177-7183.

Salinas, P. \& Contreras, A. (2003). Identification and analysis of Escherichia coli proteins that interact with the histidine kinase NtrB in a yeast two-hybrid system. Mol Gen Genomics 269, 574-581.

Sawers, R. G. (2005). Formate and its role in hydrogen production in Escherichia coli. Biochem Soc Trans 33, 42-46.

Serres, M. H., Goswami, S. \& Riley, M. (2004). GenProtEC: an updated and improved analysis of functions of Escherichia coli K-12 proteins. Nucleic Acids Res 32, D300-D302.

Silva, M. T., Sousa, J. C. F., Polonia, J. J. \& Macedo, P. M. (1979). Effects of local anesthetics on bacterial cells. J Bacteriol 137, 461-468.

Stancik, L. M., Stancik, D. M., Schmidt, B., Barnhart, D. M., Yoncheva, Y. N. \& Slonczewski, J. L. (2002). pH-dependent expression of periplasmic proteins and amino acid catabolism in Escherichia coli. J Bacteriol 184, 4246-4258.

Vijayanathan, V., Thomas, T. \& Thomas, T. J. (2002). DNA nanoparticles and development of DNA delivery vehicles for gene therapy. Biochemistry 41, 14085-14094.

Wallace, H. M. (2003). Polyamines and their role in human disease an introduction. Biochem Soc Trans 31, 354-355.

Wang, Y. D., Zhao, S. \& Hill, C. W. (1998). Rhs elements comprise three subfamilies which diverged prior to acquisition by Escherichia coli. J Bacteriol 180, 4102-4110.

Wang, D., Ding, X. \& Rather, P. N. (2001). Indole can act as an extracellular signal in Escherichia coli. J Bacteriol 183, 4210-4216.

Wright, B. E. (2004). Stress-directed adaptive mutations and evolution. Mol Microbiol 52, 643-650.

Yang, H., Wolff, E., Kim, M., Diep, A. \& Miller, J. H. (2004). Identification of mutator genes and mutational pathways in Escherichia coli using a multicopy cloning approach. Mol Microbiol 53, 283-295.

Yohannes, E., Barnhart, D. M. \& Slonczewski, J. L. (2004). pHdependent catabolic protein expression during anaerobic growth of Escherichia coli K-12. J Bacteriol 186, 192-199. 